\title{
Sn-Based Perovskite with a Wide Visible-Light Absorption Band Assisted by Hydride Doping
}

Masashi Nakamura, ${ }^{1,2}$ Hiroshi Watanabe, ${ }^{3}$ Hirofumi Akamatsu, ${ }^{3}$ Kotaro Fujii, ${ }^{1}$ Masatomo Yashima, ${ }^{1}$ George Hasegawa, ${ }^{4}$ Miki Inada,${ }^{5}$ Katsuro Hayashi, ${ }^{* 3}$ and Kazuhiko Maeda ${ }^{* 1}$

${ }^{1}$ Department of Chemistry, School of Science, Tokyo Institute of Technology, 2-12-1-NE-2 Ookayama, Meguro-ku, Tokyo, 152-8550, Japan

${ }^{2}$ Konoshima Chemical Co., Ltd., 80 Koda, Takuma, Mitoyo, Kagawa, 769-1103, Japan

${ }^{3}$ Department of Applied Chemistry, Graduate School of Engineering, Kyushu University, 744

Motooka, Nishi-ku, Fukuoka, 819-0395, Japan

${ }^{4}$ Institute of Materials and Systems for Sustainability, Nagoya University, Furo-cho, Chikusa-ku, Nagoya, 464-8603, Japan

${ }^{5}$ Center of Advanced Instrumental Analysis, Kyushu University, Kasuga-shi, Fukuoka, 816-8580, Japan

Corresponding Authors

K.H.: k.hayashi@cstf.kyushu-u.ac.jp, K.M.: maedak@chem.titech.ac.jp 

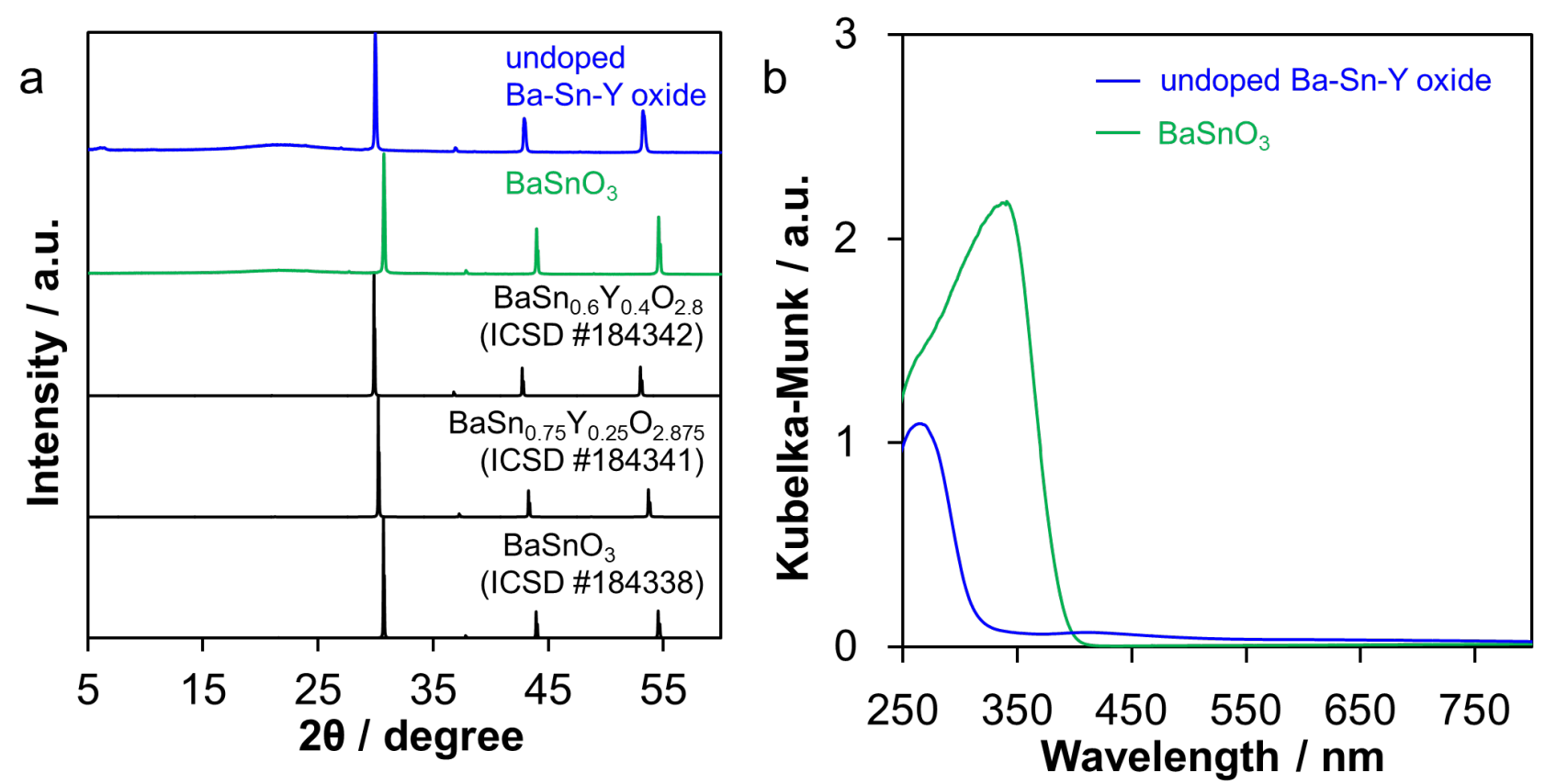

Figure S1. (a) XRD patterns and (b) UV-visible DRS spectra of undoped $\mathrm{Ba}-\mathrm{Sn}-\mathrm{Y}$ oxide and $\mathrm{BaSnO}_{3}$.

Additional Notes on UV-visible DRS. The higher concentration of oxygen vacancies in the undoped Ba-Sn-Y oxide than in $\mathrm{BaSnO}_{3}$ is suggested by an absorption tail extending to visible light region, in addition to the absorption edge at around $310 \mathrm{~nm}$. Similar absorption tailing at longer wavelengths has been reported in metal oxides having oxygen vacancies introduced by reduction with $\mathrm{H}_{2}$ gas. ${ }^{\mathrm{S} 1-3}$ Therefore, it is reasonable to conclude that the undoped $\mathrm{Ba}-\mathrm{Sn}-\mathrm{Y}$ oxide (i.e., $\mathrm{BaSn}_{0.7} \mathrm{Y}_{0.3} \mathrm{O}_{3-\delta}$ ) contained higher density of oxygen vacancies than $\mathrm{BaSnO}_{3}$.

\section{Additional Discussion on the Difference in Absorption Edge between undoped Ba-Sn-Y oxide and}

BaSnO$_{3}$. As listed in Table $\mathrm{S} 1$, the lattice constant $(a)$ of $\mathrm{BaSn}_{0.7} \mathrm{Y}_{0.3} \mathrm{O}_{3-\delta}$, calculated by the Le Bail method, was larger than that of $\mathrm{BaSnO}_{3}$. This is reasonable, considering that $\mathrm{Y}^{3+}$ having larger size than $\mathrm{Sn}^{4+}$ is partially substituted for the $\mathrm{Sn}^{4+}$ site in $\mathrm{BaSnO}_{3}$. The expansion of the crystal lattice should weaken orbital interactions among the constituent atoms, making the widths of valence and conduction bands narrower, which eventually results in a larger band gap. 

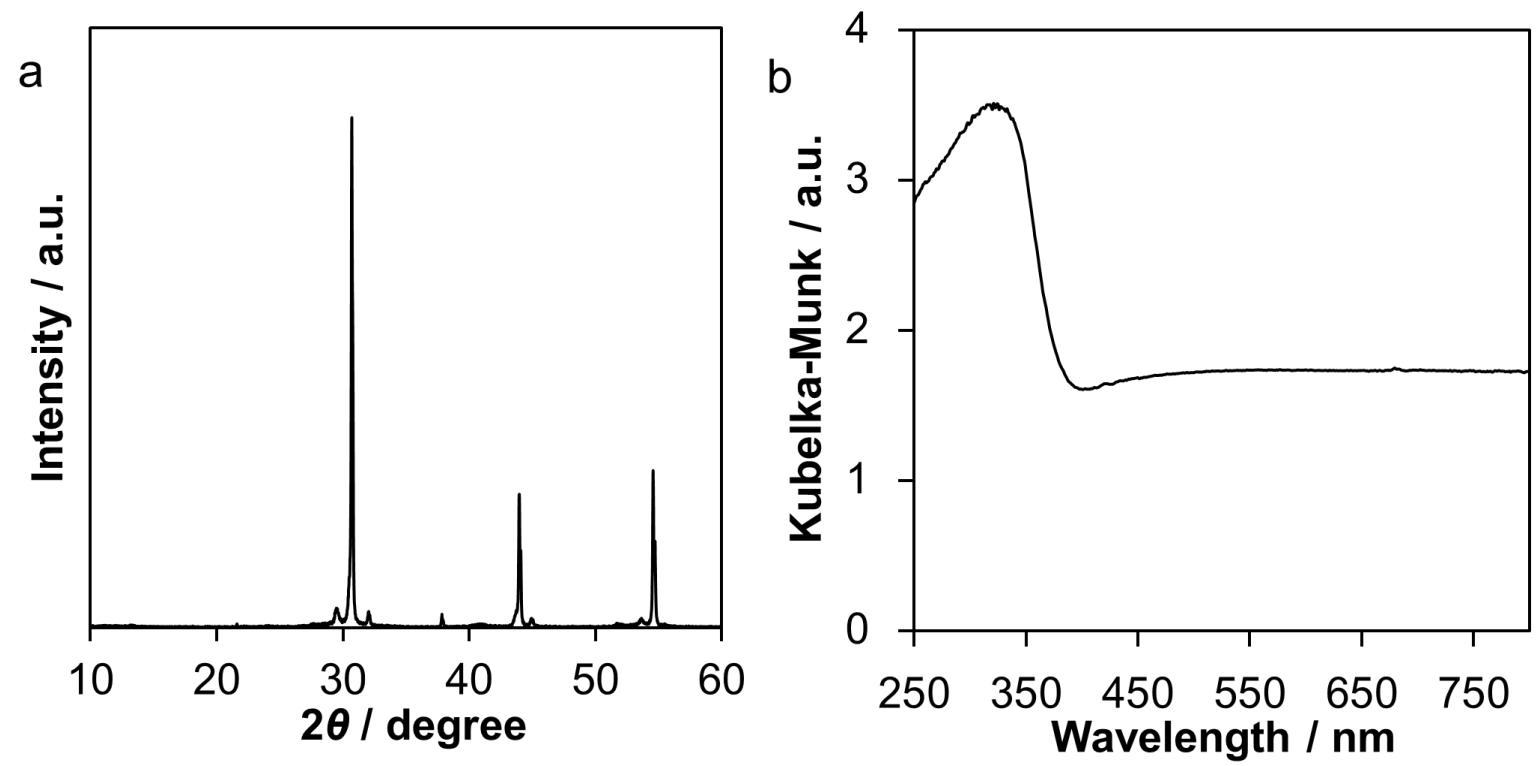

Figure S2. (a) XRD pattern and (b) UV-visible DRS spectrum of $\mathrm{CaH}_{2}$-treated $\mathrm{BaSnO}_{3}$ (without $\mathrm{Y}$ substitution).

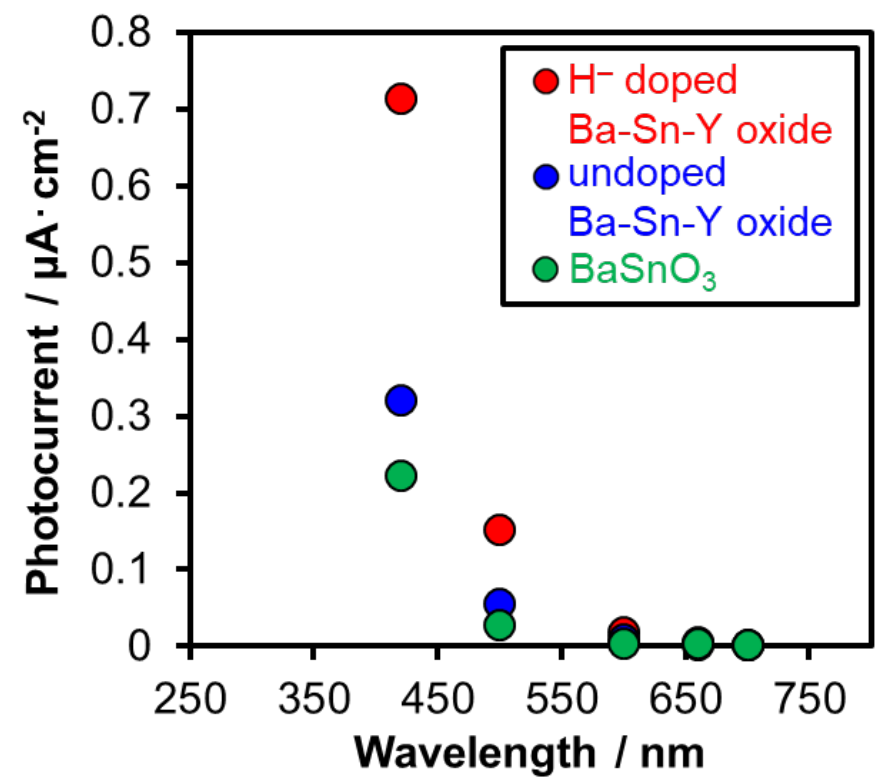

Figure S3. Wavelength-dependent photocurrent generation by undoped $\mathrm{Ba}-\mathrm{Sn}-\mathrm{Y}$ oxide and $\mathrm{BaSnO}_{3}$, along with that of $\mathrm{H}^{-}$doped $\mathrm{Ba}-\mathrm{Sn}-\mathrm{Y}$ oxide. The experimental conditions are the same as in Figure 11. Small photoresponse from undoped $\mathrm{Ba}-\mathrm{Sn}-\mathrm{Y}$ oxide and $\mathrm{BaSnO}_{3}$ under visible light is likely to originate from absorption derived from oxygen vacancies existing in these materials. 
Table S1. Lattice constants and band gaps of $\mathrm{H}^{-}$-doped, undoped $\mathrm{Ba}-\mathrm{Sn}-\mathrm{Y}$ oxides and $\mathrm{BaSnO}_{3}$.

\begin{tabular}{lll}
\hline Compound & Lattice constant $/ \AA^{a}$ & Band gap / eV $b$ \\
\hline $\mathrm{H}^{-}$-doped Ba-Sn-Y oxide & 4.2194 & 2.0 \\
undoped Ba-Sn-Y oxide & 4.2071 & 4.0 \\
$\mathrm{BaSnO}_{3}$ & 4.1156 & 3.1
\end{tabular}

${ }^{a}$ Calculated from the XRD patterns by the Le Bail method. ${ }^{b}$ Estimated from the UV-visible DRS spectra.

\section{References}

(S1) Maeda, K. Effects of the Physicochemical Properties of Rutile Titania Powder on Photocatalytic Water Oxidation. ACS Catal. 2014, 4 (6), 1632-1636.

(S2) Nishioka, S.; Hyodo, J.; Vequizo, J. J. M.; Yamashita, S.; Kumagai, H.; Kimoto, K.; Yamakata, A.; Yamazaki, Y.; Maeda, K. Homogeneous Electron Doping into Nonstoichiometric Strontium Titanate Improves Its Photocatalytic Activity for Hydrogen and Oxygen Evolution. ACS Catal. 2018, 8 (8), 7190-7200.

(S3) Amano, F.; Nakata, M.; Yamamoto, A.; Tanaka, T. Effect of $\mathrm{Ti}^{3+}$ Ions and Conduction Band Electrons on Photocatalytic and Photoelectrochemical Activity of Rutile Titania for Water Oxidation. J. Phys. Chem. C 2016, 120 (12), 6467-6474. 